\title{
Blocking Efficacy of Small Coalitions in Myopic Economies
}

\author{
Carlos Hervés-Beloso ${ }^{1}$
}

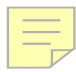

\author{
Facultad de Económicas, Universidad de Vigo, Spain \\ cherves@uvigo.es
}

\section{Emma Moreno-García ${ }^{1,2}$}

Faculdade de Economia, Universidade Nova de Lisboa, Portugal egarcia@fe.unl.pt

\section{Carmelo Núñez-Sanz ${ }^{1}$}

\author{
Departamento de Economía, Universidad Carlos III de Madrid, Spain \\ cnunez@eco.uc3m.es \\ and \\ Mário Rui Páscoa ${ }^{3}$
}

Faculdade de Economia, Universidade Nova de Lisboa, Portugal pascoa@fe.unl.pt

\begin{abstract}
We consider a continuum economy with infinitely many commodities and show that, for any positive $\varepsilon$, the core of the economy coincides with the set of allocations which are not blocked by any coalition with measure less than $\varepsilon$. Actually, our main result is an extension of Grodal's (1972, Econometrica 40, 581-583) result and, therefore, Schmeidler's (1972, Econometrica 40, 579-580) result to economies whose commodity space is $\ell^{\infty}$, for myopic preferences. Journal of Economic Literature Classification Numbers: C71, D51.

Key Words: continuum economy; coalitions; core; perfect competition; Walrasian equilibrium.
\end{abstract}

${ }^{1}$ Supported by Research Grant PB95-0729-C02 from the Dirección General de Investigación Científica y Técnica (DGICYT), Spanish Ministry of Education.

${ }^{2}$ Support from the Spanish Ministry of Education through a post doctoral fellowship in 1997 and from a Training and Mobility of Researchers (E.C.) fellowship in 1998 while visiting Universidade Nova de Lisboa, is acknowledged.

${ }^{3}$ Support from the Portuguese Ministry of Science and Technology (project Praxis PCSHC-CEG9-96), is acknowledged. 


\section{INTRODUCTION}

Eight years after the publication of Aumann's [5] core equivalence theorem, two notes in a same issue of Econometrica gave a sharper interpretation to the theorem as a characterization of perfect competition. Schmeidler [23] showed that in an atomless economy, where finitely many commodities are traded, any allocation that is not blocked by "small" coalitions is in the core. More precisely, Schmeidler [23] proved that if an allocation $f$ is blocked by a coalition $S$ via an allocation $g$, then, for any $\varepsilon>0, f$ can be blocked via the same allocation $g$ by a coalition $S^{\prime} \subset S$, with $\mu\left(S^{\prime}\right) \leqslant \varepsilon$. Grodal [12] showed that we can further restrict the set of coalitions, to those consisting of finitely many arbitrarily small sets of agents with similar characteristics, which are presumably easier to form and also to interpret. Precisely, Grodal [12] established that an allocation belongs to the core if and only if it cannot be blocked by a coalition which is the union of at most $\ell+1$ subcoalitions, each of which has measure and diameter less than $\varepsilon$, where $\ell$ is the number of commodities to be exchanged in the market. These results imply that, for finite-dimensional commodity spaces, the set of Walrasian allocations of an atomless economy coincides with the set of allocations that are unblocked by arbitrarily small coalitions.

The relation between the core and the Walrasian allocations set has been studied in the context of infinite dimensional commodity spaces by many authors (see Bewley [7], Ostroy [20], Mas-Colell [16], and Ostroy-Zame [21], on core equivalence, and Anderson-Zame [2,3] on core convergence). In this context the relation between the two allocation sets becomes even more interesting since the diversity in agents' preferences and endowments is now potentially higher and, therefore, blocking may become more difficult, as illustrated by recent examples of core-inequivalence and failure of core convergence (see Ostroy-Zame [21] and Anderson-Zame [2,3], respectively). It becomes also interesting to examine whether the above epsilon-core equivalence results are extendable to infinite dimensional commodity spaces.

Schmeidler's [23] and Grodal's [12] results rely heavily on Liapunov's convexity theorem, which does not hold in an infinite dimensional set up. This implies that an immediate extension of these results to atomless economies with an infinite dimensional commodity space is not possible. That is, the exact version of Schmeidler's result does not hold for atomless economies with an infinite dimensional commodity space and, therefore, in such economies the exact version of Grodal's result does not hold either. C. Núñez [19] gave an example of an atomless economy, with infinitely many commodities, where an allocation $f$ is blocked by the coalition of all agents via an allocation $g$, but there is no other different coalition blocking $f$ via the same allocation $g$. 
Despite this impossibility for obtaining both results in their exact strong versions, we were able to find an extension of the theorem stated in Grodal [12] to atomless economies with an infinite dimensional commodity space. Precisely, we show that in continuum economies whose commodity space is $\ell^{\infty}$, the space of bounded sequences, the following property holds: if an allocation $f$ is blocked by a coalition via an allocation $g$, then there exists a natural number $N=N(f)$ and an allocation h, such that, for any $\varepsilon>0$, $f$ can be blocked via $h$ by a subcoalition which is the union of at most $N$ sets, each of which has measure and diameter less than $\varepsilon$. As a consequence, in a continuum economy with infinitely many commodities, it is enough to consider the blocking power of arbitrarily small coalitions in order to obtain the core. Thus, we also extend Schmeidler's [23] result to the infinite dimensional setting. For this, we assume that preferences are Mackey continuous.

Existence and core equivalence of equilibria for the commodity space $\ell^{\infty}$ (with prices in $\ell_{1}$ ) were addressed by Bewley $[6,7]$, under the assumptions of convexity, monotonicity and Mackey continuity of preferences. Mackey upper semi-continuous (usc) preferences are known to be upper myopic, in the sense that gains in the distant future are negligible (see also BrownLewis [8] and Araujo [4]). Our extension of Schmeidler's [23] and Grodal's [12] results uses also the Mackey lower semi-continuity (lsc) of preferences, which corresponds to lower myopia: losses in the distant future are negligible (in the terminology of Mas-Colell and Zame [18], example 4.1). Roughly speaking, lower myopia allows us to drop tails from the blocking allocation and, subsequently, use Grodal's [12] finite-dimensional approach. Under Bochner integrability of the endowments allocation the argument can be carried out uniformly on agents and this is done by passing first to a compact blocking subcoalition where preferences, blocking allocation and blocked allocation are continuous, with respect to the agents.

Bochner integrability of the endowments allocation was identified by Gretsky-Ostroy [11] with physical thickness of markets. Our result asserts that, for the space $\ell^{\infty}$, under Mackey continuity of preferences and Bochner integrability of endowments, it is not possible to find outside the Walrasian set, imperfectly competitive outcomes where the imperfection of competition is exercised only by coalitions of arbitrarily small size (since these outcomes should belong to an $\varepsilon$-core) More work is needed to infer that the result still holds for other commodity spaces, namely for the space of measures, where the Walrasian allocations are known to resist manipulation by arbitrarily small coalitions (see Ostroy-Zame [21] and Hervés-Moreno-Páscoa [14]) and may, therefore, be regarded as true perfectly competitive outcomes.

Presumably, if the commodity spaces were other duals of separable Banach spaces, we would need to strengthen the Mackey continuity to 
weak star continuity on preferences. Weak star continuity of preferences, defined on duals of separable Banach spaces, has been extensively used in the perfect competition literature, namely by Mas-Colell [16], Hart [13], Jones [15] Gretsky-Ostroy [11] and Ostroy-Zame [21], and was related to economic thickness of markets in the last two articles.

The remainder of this paper is organized as follows. In section 2, we formalize the model and state the assumptions. In section 3, we extend Grodal's [12] result to continuum economies with infinitely many commodities. In section 4 , we present some examples. The first example shows that the exact strong versions of the results in Schmeidler [23] and in Grodal [12] do not hold, in general, when infinitely many commodities are traded. The second example shows that, if preferences are not Mackey continuous, then our main result does not hold either.

\section{THE MODEL}

Let us consider a pure exchange economy $\mathscr{E}=\left((I, \mathscr{A}, \mu), X_{t}, \omega(t), \succcurlyeq_{t}, t \in I\right)$, with a continuum of agents and $\ell_{+}^{\infty}$ as its commodity space. $(I, \mathscr{A}, \mu)$ is an atomless positive, bounded measure space which represents the space of agents. For simplicity, we assume that $I$ is the real interval $[0,1], \mathscr{A}$ is the Lebesgue $\sigma$-algebra of subsets of $I$, and $\mu$ is the Lebesgue measure. Each agent $t \in I$ is characterized by her consumption set $\ell_{+}^{\infty}$, her initial endowment $\omega(t)=\left(\omega_{j}(t)\right)_{j=1}^{\infty} \in \ell_{+}^{\infty}$, and her preference relation $\succcurlyeq_{t}$ on $\ell_{+}^{\infty} \times \ell_{+}^{\infty}$.

For any $x \in \ell^{\infty}, y \in \ell_{1}$, let $\langle x, y\rangle=\sum_{j=1}^{\infty} x_{j} y_{j}$.

A function $f: I \rightarrow \ell^{\infty}$ is Gelfand integrable iff for each $p \in \ell_{1}$, there is $x \in \ell_{\infty}$ such that, the real valued function $t \rightarrow\langle p, f(t)\rangle$ is Lebesgue integrable and $\int_{I}\langle p, f(t)\rangle d \mu(t)=p \cdot x$. We say that $x$ is the Gelfand integral of $f$ on $I$, and we write $\oint_{I} f(t) d \mu(t)=x$.

A function $f: I \rightarrow \ell^{\infty}$ is strongly measurable if there exists a sequence of simple functions $\left(f_{n}\right)$ such that $\lim _{n \rightarrow \infty}\left\|f_{n}(t)-f(t)\right\|=0$. A measurable function is said to be Bochner integrable if there exists a sequence of simple functions $\left(f_{n}\right)$ such that $\lim _{n \rightarrow \infty} \int_{I}\left\|f_{n}(t)-f(t)\right\| d \mu(t)=0$. In this case, for each measurable set $S \subset I$, we define $\int_{S} f(t) d \mu(t)=\lim _{n \rightarrow \infty} \int_{S} f_{n}(t) d \mu(t)$. It can be easily shown that if $f$ is Bochner integrable then $f$ is Gelfand integrable and the integrals coincide. We remark that if $f$ is measurable, then $f$ is Bochner integrable iff $\int_{I}\|f(t)\| d \mu(t)<\infty$. (See Diestel and Uhl [9], p. 45).

As in Bewley [7], an allocation is a Gelfand integrable function $f: I \rightarrow \ell_{+}^{\infty}$. However, we will impose the stronger integrability condition on the endowment allocation: 
(H.1) The map $\omega: I \rightarrow \ell_{+}^{\infty}$, which associates to each agent her initial endowment, is Bochner integrable.

\section{Moreover,}

(H.2) For every measurable set $A \subset I$, with $\mu(A)>0$, there exists a real number $a=a(A)>0$, such that $\int_{A} \omega_{j}(t) d \mu(t) \geqslant a$ for all $j$.

Remark 1. Araujo [4] studied the existence of equilibrium and Pareto optimal allocations in economies with an infinite number of commodities but with a finite number of agents. For this, he stated an assumption about boundedness of endowments, whose natural extension for the case of a continuum economy is the next condition: $(\mathrm{H}) \omega_{j}(t) \geqslant a(t)>0$, for all $j$, for almost all $t \in I$.

Observe that if $\omega$ satisfies $(\mathrm{H})$, then $\omega$ also verifies our condition (H.2). However, condition $(\mathrm{H})$ is stronger than our assumption (H.2). For this, let $J$ be the set of rational numbers in the interval $(0,1)$, that is, $J=(0,1) \cap \mathbb{Q}=\left\{q_{j} \mid j \in \mathbb{N}\right\}$. Consider $\omega$, defined by $\omega_{j}(t)=\left|t-q_{j}\right|, q_{j} \in J$. Then, $\inf _{j}\left\{\omega_{j}(t)\right\}=0$, for every $t \in I$. It is not hard to prove that $\int_{A} \omega_{j}(t) d \mu(t) \geqslant(\mu(A))^{2} / 4$, whatever positive measure set $A$ may be.

We will assume that preference relations are Mackey continuous. Let $\mathscr{C}_{*}\left(\ell_{+}^{\infty}\right)$ denote the space of Mackey continuous functions on $\left(\ell_{+}^{\infty}\right)$. Since $\ell^{\infty}$, endowed with the Mackey topology is separable (see Appendix), preference relations will be representable by Mackey continuous utility functions. Actually, we will impose the following measurability condition on the representations:

(H.3) The mapping $U: I \rightarrow \mathscr{C}_{*}\left(\ell_{+}^{\infty}\right)$, which associates to each agent $t$ an utility function $U(t)=U_{t}$ representing her preference relation is measurable (with respect to the topology of uniform convergence on bounded sets of $\left.\ell_{+}^{\infty}\right)$.

Remark 2. The above condition would follow easily from a primitive measurability assumption on preferences if the consumption set were weak star compact. Suppose the consumption set were $M=\left\{x=\left(x_{n}\right)_{n=1}^{\infty} \in\right.$ $\ell_{+}^{\infty} \mid x_{n} \leqslant H$ for all $\left.n\right\}$ for some positive scalar H. On $M$ the Mackey and the weak star topology coincide (see Appendix). Let $\mathscr{P}$ denote the set of Mackey continuous preference relations. We remark that $\mathscr{P}$ is a subspace of the space of nonempty weak star closed subsets of $M \times M$. We consider on $M \times M$ the closed convergence topology. The standard assumption is that the mapping $P: I \rightarrow \mathscr{P}$, which associates to each agent $t$ her preference relation is measurable. As $M$, endowed with the Mackey topology (equivalently, with the weak star topology), is compact and separable, there 
exists a continuous function $\mathscr{U}: \mathscr{P} \times M \rightarrow \mathbb{R}$, such that $\mathscr{U}(\succcurlyeq, \cdot)$ is an utility function which represents the preference relation $\geqslant \in \mathscr{P}$ (see Mas-Colell [17]). Let $\mathscr{C}(M)$ denote the set of all real bounded continuous functions defined on $M$ and endow $\mathscr{C}(M)$ with the norm topology. Then, the function $\mathscr{V}: \mathscr{P} \rightarrow \mathscr{C}(M)$, given by $\mathscr{V}(\geqslant)=\mathscr{U}(\geqslant, \cdot)$, is a continuous function (see Wilansky [24, theorem 13.3.4]). Therefore, the composition of $P$ with $\mathscr{V}$, given by $U: I \rightarrow \mathscr{C}(M)$, is measurable.

A preference relation $\geqslant$ is weakly monotone if $x=\left(x_{n}\right), y=\left(y_{n}\right)$ and $x_{n} \geqslant y_{n}$ for every $n$, then $x \geqslant y$.

(H.4) We also assume that the preference relation $\succcurlyeq_{t}$ is weakly monotone for almost all $t \in I$.

We will recall other monotonicity concepts which will be referred to when discussing work by other authors. Given a preference relation $\succcurlyeq$, denote $x>y$ if $x \geqslant y$ but not $y \geqslant x$. A preference relation is said to be monotone if $x \gg y\left(x_{n}>y_{n}\right.$ for every $\left.n\right)$, implies $x>y$. This assumption was used in Bewley's $[6,7]$ existence and core equivalence theorems. Monotonicity is actually implied by a stronger version of monotonicity, contemplated by Brown-Lewis [8]: $x>y\left(x_{n} \geqslant y_{n}\right.$ for every $n$, and $x_{i}>y_{i}$ for some $i$ ) implies $x>y$.

Remark 3. Mackey usc of preferences implies upper myopia: given any bundle $z$, let $z^{(n)}$ be the bundle defined by $z_{j}^{(n)}=0$ for $j \leqslant n$ and $z_{j}^{(n)}=z_{j}$ otherwise. Then, $x>y$ implies $x>y+z^{(n)}$ for $n$ large enough, since $z^{(n)}$ converges to $z$ in the Mackey topology (see Aliprantis and Border [1, Corollary 13.29]). Slightly different concepts of upper myopia are discussed by Brown-Lewis [8] and Araujo [4], all coinciding when preferences are monotonic. It is well known that the Mackey topology is the strongest topology with respect to which a continuous complete preorder is upper myopic (this fact was shown by Bewley [6], under monotonicity; Hildenbrand is quoted by Bewley for having suggested it). Mackey continuity of preferences was shown by Araujo [4] to be a necessary condition for existence of individually rational Pareto allocations and, therefore, for existence of Walrasian equilibria. Mackey lsc implies lower myopia: given $x>y$ and given $z$, then $x-z^{(n)}>y$ for $n$ large enough.

An allocation $f$ is feasible if $\oint_{I} f(t) d \mu(t) \leqslant \int_{I} \omega(t) d \mu(t)$.

A coalition of agents is a measurable set $S \subset[0,1]$, such that $\mu(S)>0$. A coalition $S$ blocks an allocation $f$ if there exists another allocation $g$ such that $\oint_{S} g(t) d \mu(t) \leqslant \int_{S} \omega(t) d \mu(t)$, and $g(t) \succ_{t} f(t)$ for almost all $t \in S$. The core of the economy $\mathscr{E}$, denoted by $\operatorname{Core}(\mathscr{E})$, is the set of all feasible allocations that can not be blocked by any coalition of agents. 


\section{RESULTS}

In the case of considering $\mathbb{R}^{\ell}$ as commodity space, Schmeidler [23] shows that, for arbitrary $\varepsilon>0$, it is enough to consider only coalitions with measure less than $\varepsilon$, in order to obtain the core allocations. Moreover, we can further restrict the coalitions that are allowed to form. In fact, Grodal [12] shows that an allocation belongs to the core if and only if it can not be blocked by a coalition which is the union of at most $\ell+1$ coalitions, each of which has measure and diameter less than $\varepsilon$. Our aim is to obtain an extension of these results to continuum economies with infinitely many commodities. For this, we state a preliminary result.

Proposition 1. Let $f$ be a feasible allocation in the economy $\mathscr{E}$, such that $f \notin$ Core $(\mathscr{E})$. Then, there exists a compact positive measure subset $S$ of I such that $S$ blocks $f$ via an allocation $g$ and the functions $f, g$ and $U$ are continuous on $S$.

Proof. Let $f \notin \operatorname{Core}(\mathscr{E})$. Then, there exists a coalition $A$ and an allocation $g$, such that $\oint_{A} g(t) d \mu(t) \leqslant \int_{A} \omega(t) d \mu(t)$, and $U_{t}(g(t))>U_{t}(f(t))$ for every $t \in A$. By hypothesis, there exists a positive real number $a$, such that $\int_{A} \omega_{j}(t) d \mu(t) \geqslant a$, for all $j$. Then, without lost of generality, by (H.2) and (H.4), we can consider that $\oint_{A} g_{j}(t) d \mu(t) \geqslant a$, for all $j$. This implies that there exists $B \subset A$, with $\mu(B)>0$, such that $g_{j}(t) \geqslant a$, for all $t \in B$.

For each positive integer $m$ let $\hat{g}^{m}$ be the allocation defined by $\hat{g}_{j}^{m}(t)=\left(g_{j}(t)-1 / m\right)^{+}=\max \left\{0, g_{j}(t)-1 / m\right\}$. Now, the sequence $\hat{g}^{m}(t)$ converges to $g(t)$ a.e. for the Mackey topology. To see this, recall that Mackey convergence is uniform convergence on convex weakly compact subsets of $\ell_{1}$. By Banach's theorem (see Appendix) a weakly compact subset $L$ of $\ell_{1}$ is also norm compact. Then,

$$
\begin{aligned}
\left|\left\langle g(t)-\hat{g}^{m}(t), y\right\rangle\right| & \leqslant \sum_{\left\{j: g_{j}(t) \leqslant 1 / m\right\}}\left|g_{j}(t) y_{j}\right|+\frac{1}{m} \sum_{\left\{j: g_{j}(t)>1 / m\right\}}\left|y_{j}\right| \\
& \leqslant E\left(\sum_{\left\{j: g_{j}(t) \leqslant 1 / m\right\}} g_{j}(t)+\frac{1}{m}\right)
\end{aligned}
$$

for some $E>0$ such that $\|y\|_{1} \leqslant E$ for every $y \in L$. The expression inside brackets obviously tends to zero as $m$ goes to $\infty$ and, therefore, $\left\langle\hat{g}^{m}(t), y\right\rangle$ converges to $\langle g(t), y\rangle$ a.e. uniformly on $y \in L$. Then, by Mackey continuity of preferences, $U_{t}\left(\hat{g}^{m}(t)\right)$ converges to $U_{t}(g(t))$ almost everywhere.

By Lusin's theorem (see Bewley [7]), there exists a compact set $K_{1} \subset[0,1]$, with $\mu\left(K_{1} \cap B\right)>0$, such that the allocations $g$ and $f$ are continuous on $K_{1}$, with respect the weak star topology on $\ell_{+}^{\infty}$, and the mapping $U$ is also continuous on $K_{1}$ (with respect to the topology of uniform 
convergence on bounded subsets of $\left.\ell_{+}^{\infty}\right)$. Actually, $f$ and $g$ are Mackey continuous on $K_{1}$, since the weak star continuity of $f$ and $g$ implies that $f\left(K_{1}\right)$ and $g\left(K_{1}\right)$ are bounded subsets of $\ell_{+}^{\infty}$ and the corollary in Appendix applies.

Thus, by Egoroff theorem, there exists a compact $K_{2}$, such that $U_{t}\left(\hat{g}^{m}(t)\right)$ converges to $U_{t}(g(t))$ uniformly on $K_{2}$, and $\mu\left(K_{1} \cap K_{2} \cap B\right)>0$. Let $K=K_{1} \cap K_{2} \cap B$. For each positive integer $m$, consider the allocation $\tilde{g}^{m}$, given by

$$
\tilde{g}_{j}^{m}(t)= \begin{cases}\hat{g}_{j}^{m}(t) & \text { if } \quad t \in K \\ g_{j}(t) & \text { if } \quad t \notin K .\end{cases}
$$

By construction, there exists $\bar{m}$, such that $U_{t}\left(\tilde{g}^{m}(t)\right)>U_{t}(f(t))$, for all $m \geqslant \bar{m}$. Moreover, $\oint_{A} \tilde{g}^{m}(t) d \mu(t) \ll \int_{A} \omega(t) d \mu(t)$. In fact, for all $j$ it is verified that $\oint_{A} \tilde{g}_{j}^{m}(t) d \mu(t)+\delta \leqslant \int_{A} \omega_{j}(t) d \mu(t)$, for all $\delta \leqslant \mu(K) / m$, with $m \geqslant \max \{\bar{m}, 1 / a\}$. Therefore, coalition $A$ blocks $f$ via an allocation $g^{*}$, such that $\oint_{A} g_{j}^{*}(t) d \mu(t)+\delta \leqslant \int_{A} \omega_{j}(t) d \mu(t)$, for all $j$, with $\delta>0$.

By Lusin's theorem, for each positive integer $n$, there exists a compact set $K_{n}$ with $\mu\left(A \backslash K_{n}\right) \leqslant 1 / n$, such that $U$ is continuous on $K_{n}$ and $g^{*}, f$ are Mackey continuous on $K_{n}$ (by the same argument as before). On the other hand, $\left.\int_{K_{n}} \omega_{j}(t) d \mu(t)\right)$ converges to $\int_{A} \omega_{j}(t) d \mu(t)$, uniformly on $j$. That is so, because, by (H.1), $\omega: I \rightarrow \ell_{+}^{\infty}$ is a Bochner integrable function, and then it is verified that

$$
\begin{aligned}
\sup _{j} \mid & \int_{K_{n}} \omega_{j}(t) d \mu(t)-\int_{A} \omega_{j}(t) d \mu(t) \mid \\
& \leqslant \sup _{j}\left|\int_{A \backslash K_{n}} \omega_{j}(t) d \mu(t)\right|=\left\|\int_{A \backslash K_{n}} \omega(t) d \mu(t)\right\|_{\infty} \\
& \leqslant \int_{A \backslash K_{n}}\|w(t)\|_{\infty} d \mu(t) .
\end{aligned}
$$

The last inequality is due to the fact that the function given by $t \rightarrow\|\omega(t)\|_{\infty}$ is Lebesgue integrable, because $\omega$ is a Bochner integrable function. Then, there exists $\bar{n}$, such that for all $j$, it is verified that $\mid \int_{K_{n}} \omega_{j}(t) d \mu(t)-$ $\int_{A} \omega_{j}(t) d \mu(t) \mid<\delta$, for all $n \geqslant \bar{n}$. So, the following inequalities hold:

$$
\int_{K_{n}} g^{*}(t) d \mu(t) \leqslant \oint_{A} g^{*}(t) d \mu(t) \leqslant \int_{K_{n}} \omega(t) d \mu(t), \text { for all } n \geqslant \bar{n} .
$$

Therefore, coalition $K_{n}$ blocks $f$ via $g^{*}$, for all $n$ large enough.

Q.E.D

Now we prove the main result of this paper. For this, we need some notation. Given $x=\left(x_{h}\right)_{h=1}^{\infty} \in \ell^{\infty}$ and $n \in \mathbb{N}$, we denote by $x^{n}$ the element of $\ell^{\infty}$ defined by $x_{h}^{n}=x_{h}$ if $1 \leqslant h \leqslant n$ and $x_{h}^{n}=0$ if $h>n$. 
THEOREM 1. Let $\mathscr{E}=\left((I, \mathscr{A}, \mu), X_{t}=\ell_{+}^{\infty}, \omega(t), \preccurlyeq{ }_{t}, t \in I\right)$ a pure exchange economy satisfying assumptions (H.1) through (H.4). If a feasible allocation $f$ is blocked by a coalition $D$ via an allocation $g$, then there exists a positive integer $N=N(f)$, and an allocation $h$, such that for any $\varepsilon>0$, the allocation $f$ can be blocked via $h$ by a coalition $S=\bigcup_{i=1}^{N} S_{i} \subset D$ with $\operatorname{diam}\left(S_{i}\right) \leqslant \varepsilon$ and $\mu\left(S_{i}\right) \leqslant \varepsilon$.

Proof. Let $f \notin \operatorname{Core}(\mathscr{E})$. Then, there exists a coalition $D$ and an allocation $g$, such that $\oint_{D} g(t) d \mu(t) \leqslant \int_{D} \omega(t) d \mu(t)$, and $U_{t}(g(t))>U_{t}(f(t))$ for every $t \in D$. By the previous proposition, we can take $D$ compact, $U$ continuous on $D$ and $g, f$ continuous functions on $D$ (for the Mackey topology). So, there exists $\delta>0$, such that $U_{t}\left((g(t))-\delta>U_{t}((f(t))+\delta\right.$ for every $t \in D$.

Let us show that the sequence $g^{n}$ converges to $g$ almost everywhere for the Mackey topology. To see this, let $L$ be a weakly compact subset of $\ell_{1}$. So, by Banach's theorem (see Appendix), $L$ is also a norm compact subset of $\ell_{1}$. Then, for any $\varepsilon>0$ there exists $n$ such that $\sum_{j=n}^{\infty}\left|y_{j}\right| \leqslant \varepsilon$ for all $y \in L$ (see Aliprantis and Border [1, Theorem 13.24]). Hence,

$$
\left|\left\langle g(t)-g^{n}(t), y\right\rangle\right|=\sum_{j=n}^{\infty}\left|g_{j}(t) y_{j}\right| \leqslant\|g\|_{\infty} \sum_{j=n}^{\infty}\left|y_{j}\right| \leqslant\|g\|_{\infty} \varepsilon .
$$

Therefore, $\left\langle g^{n}(t), y\right\rangle$ converges to $\langle g(t), y\rangle$ a.e. uniformly on $y \in L$. That is, $g^{n}$ converges to $g$ almost everywhere for the Mackey topology.

Now, let us see that $U_{t}\left(g^{n}(t)\right)$ converges to $U_{t}(g(t))$ uniformly on $t \in D$. For this, given $x \in \ell_{+}^{\infty}$, let $\left(x^{k}\right)$ be a sequence Mackey converging to $x$. Let $t_{n}$ a sequence converging to $t \in D$. Then, $\lim _{k \rightarrow \infty} U_{t_{n}}\left(x^{k}\right)=U_{t_{n}}(x)$ for all $n$, and $\lim _{n \rightarrow \infty} U_{t_{n}}\left(x^{k}\right)=U_{t}\left(x^{k}\right)$ for all $k$. Moreover, as $U$ is continuous on $D$, this last convergence is uniform on $k$ ( since $U_{t_{n}}(\cdot) \rightarrow U_{t}(\cdot)$ on $\mathscr{C}_{*}\left(\ell_{+}^{\infty}\right)$, for the topology of uniform convergence on bounded sets and, by Alaoglu theorem, the sequence $\left(x^{k}\right)$ is bounded, since it is Mackey convergent and, therefore, weak star convergent). Applying Moore's lemma (see DunfordSchwartz [10, I.7.6]), $\lim _{n \rightarrow \infty} \lim _{k \rightarrow \infty} U_{t_{n}}\left(x^{k}\right)=\lim _{k \rightarrow \infty} \lim _{n \rightarrow \infty} U_{t_{n}}\left(x^{k}\right)$ $=U_{t}(x)$. Now, since $g: D \rightarrow \ell_{+}^{\infty}$ is a Mackey continuous function and $g^{n}(t)$ Mackey converges to $g(t)$ for almost all $t \in I=[0,1]$, we have $\lim _{n \rightarrow \infty} U_{t_{n}}\left(g^{n}\left(t_{n}\right)\right)=U_{t}(g(t))$. That is, $U(\cdot)\left(g^{n}(\cdot)\right)$ converges continuously to $U(\cdot)(g(\cdot))$. Equivalently, $U(\cdot)\left(g^{n}(\cdot)\right)$ converges to $U(\cdot)(g(\cdot))$ uniformly on compact subsets of $D$. (See Royden [22, Problem 9.40]).

In this way, we obtain that there exists $N$, such that for all $t \in D$, it is verified that $U_{t}\left(g^{N}(t)\right)>U_{t}(g(t))-\delta>U_{t}(f(t))+\delta$, where the first inequality follows by Mackey continuity of preferences. Then, coalition $D$ blocks $f$ via the allocation $g^{N}$. We have the following inequality between 
$N$-dimensional Lebesgue integrals, $\int_{D} g^{N}(t) d \mu(t) \leqslant \int_{D} \omega^{N}(t) d \mu(t)$. Applying now Grodal's [12] N-dimensional argument, we obtain that for any $\varepsilon>0$, there exists a coalition $S=\bigcup_{i=1}^{N} S_{i} \subset D$ with $\operatorname{diam}\left(S_{i}\right) \leqslant \varepsilon$ and $\mu\left(S_{i}\right) \leqslant \varepsilon$, such that $S$ blocks $f$ via $g^{N}$, since $\int_{S} g^{N}(t) d \mu(t) \leqslant$ $\int_{S} \omega^{N}(t) d \mu(t)<\int_{S} \omega(t) d \mu(t)$.

Q.E.D

Remark 4. We have proved that in an atomless economy with infinitely many commodities the following property holds: if an allocation $f$ is blocked by a coalition via an allocation $g$, then for any $\varepsilon>0$, it can be blocked via another allocation $h$ by a coalition which is the union of at most $N=N(f)$ coalitions, each of which has measure and diameter less than $\varepsilon$. In particular, we have proved that, in a continuum economy with infinitely many commodities, it is enough to consider arbitrarily small coalitions, in order to obtain the core. The result obtained by Grodal [12] in the finite-dimensional case is stronger: in an atomless economy with a finite number of commodities, if an allocation $f$ is blocked by a coalition via an allocation $g$, then for any $\varepsilon>0$, it can be blocked via the same allocation $g$ by a coalition which is the union of $N$ coalitions, each of which has measure and diameter less than $\varepsilon$. In Grodal's [12] corollary, $\mathbf{N}$ is independent of $\mathrm{f}$ and is actually equal to $\ell$, where $\ell$ is the number of commodities in the economy.

\section{SOME EXAMPLES}

EXAMPLE 1. This first example shows that the exact strong version of Schmeidler's [23] result cannot be true, in general, for an infinite-dimensional commodity space. That is, if $S$ blocks $f$ via $g$ it is in general impossible to find, for each $\varepsilon>0$, a subcoalition with measure not exceeding $\varepsilon$ and still blocking $f$ via the same alternative allocation $g$. Then, the result in Grodal [12], as it is written in Remark 4, does not hold either, in general, when there are infinitely many commodities. The example was inspired by Núñez's [19] observation, although it is constructed differently.

Consider a pure exchange economy $\mathscr{E}=\left((I, \mathscr{A}, \mu), \omega(t), U_{t}(\cdot), t \in I\right)$, with $\ell^{\infty}$ as commodity space. For each $j \in \mathbb{N}$, let $n(j), h(j) \in \mathbb{N} \cup\{0\}$, such that $2^{n(j)} \leqslant j<2^{n(j)+1}$, and $h(j)=j-2^{n(j)}$. The map $\omega: I \rightarrow \ell_{+}^{\infty}$ that associates to each agent $t \in I$ her initial endowment $\omega(t)$ is given by:

$$
\omega_{j}(t)= \begin{cases}\frac{1}{2}+C(j) & \text { if } t \in B(j)=\left(\frac{h(j)}{2^{n(j)}}, \frac{h(j)+1}{2^{n(j)}}\right) \\ \frac{1}{2} & \text { in the other case, }\end{cases}
$$


where $C(j)>0$ verifies that $C(j)=C\left(j^{\prime}\right)$ if $n(j)=n\left(j^{\prime}\right)$, and $C(j)$ converges to 0 when $j$ goes to $\infty$. This last property implies that $\omega$ is essentially bounded and Bochner integrable.

Every agent has the same preference relation, represented by the following utility function

$$
U(x)=\sum_{j \geqslant 1} \alpha(j) \log \left(\frac{1}{2}+x_{j}\right)
$$

where $\alpha(j)>0$ verifies that $\alpha(j)=\alpha\left(j^{\prime}\right)$ if $n(j)=n\left(j^{\prime}\right)$, and $\sum_{j \geqslant 1} \alpha(j)<\infty$.

Let $\alpha_{n}=\alpha(j)$, when $n(j)=n$. It is important to notice that for every agent $t \in I$ it is verified that $U(\omega(t)) \leqslant \sum_{n \geqslant 0} \alpha_{n} \log \left(1+C_{n}\right)$, with strict inequality if $t \in \mathbb{D}=\left\{k / 2^{m}, m \in \mathbb{N}, k=1, \ldots, 2^{m}-1\right\}$, and equality in other case.

The coalition $I$ blocks $\omega$ via the allocation $x$ given by $x_{j}(t)=$ $x_{j}=1 / 2+\left(C(j) / 2^{n(j)}\right)$. That is so because for all $t \in I$ it is verified that

$$
\begin{aligned}
U(x(t)) & =\sum_{n \geqslant 0} \alpha_{n}\left(\sum_{2^{n} \leqslant j<2^{n+1}} \log \left(1+\frac{C_{n}}{2^{n}}\right)\right)=\sum_{n \geqslant 0} \alpha_{n} \log \left(1+\frac{C_{n}}{2^{n}}\right)^{2^{n}} \\
& >\sum_{n \geqslant 0} \alpha_{n} \log \left(1+C_{n}\right) \geqslant U(\omega(t)) .
\end{aligned}
$$

The strict inequality is due to the fact that $\left(1+C_{n} / 2^{n}\right)^{2^{n}}>1+C_{n}$, because $\left(1+C_{n} / k\right)^{k}>1+C_{n}$, for all $k$.

Let $S$ be a coalition, such that $0<\mu(S)<1$. Let us see that $S$ can not block $\omega$ via the same allocation $x$. In fact, we claim that the inequality $\int_{S} x(t) d \mu(t) \leqslant \int_{S} \omega(t) d \mu(t)$ does not hold. To prove our point, assume that $\int_{S} x(t) d \mu(t) \leqslant \int_{S} \omega(t) d \mu(t)$. Then, if we take $j>1,2^{n} \leqslant j<2^{n+1}$, we obtain that

$\mu(S)\left(\frac{1}{2}+\frac{C(j)}{2^{n(j)}}\right)=\int_{S} x_{j}(t) d \mu(t) \leqslant \int_{S} \omega_{j}(t) d \mu(t)=C(j) \mu\left(S \cap B_{j}\right)+\frac{1}{2} \mu(S)$.

These $2^{n}$ inequalities imply that $\left(\mu(S) / 2^{n(j)}\right) \leqslant \mu\left(S \cap B_{j}\right)$. Let $n(j)=n$, $h(j)=i$. Then, we obtain that $\mu(S) / 2^{n(j)} \leqslant \mu\left(S \cap\left(i / 2^{n},(i+1) / 2^{n}\right)\right)$, with $i=0, \ldots, 2^{n}-1$. Let us show that this can not hold for all $i$ and $n$. For this, consider the function $F(\bar{t})=\int_{0}^{\bar{t}} \chi_{S}(t) d \mu(t)$. By Lebesgue differentiation theorem, we have that there exists $B \subset I$ with $\mu(B)=0$, such that $F^{\prime}(t)=\chi_{S}(t)$, for all $t \in I \backslash B$. Let $t_{0} \in I \backslash(S \cup \mathbb{D} \cup B)$, and let $a_{m}, b_{m}>0$, such that

$t_{0}-b_{m}=\frac{k_{m}}{2^{m}}<t_{0}<t_{0}+a_{m}=\frac{\left(k_{m}+1\right)}{2^{m}}, \quad$ with $m \in \mathbb{N}, k_{m} \in\left\{0, \ldots, 2^{m}-1\right\}$. 
Then, it is verified that

$$
F^{\prime}\left(t_{0}\right)=\lim _{m \rightarrow \infty} \frac{F\left(t_{0}+a_{m}\right)-F\left(t_{0}-b_{m}\right)}{a_{m}+b_{m}}=\lim _{m \rightarrow \infty} \frac{\mu\left(\left(\frac{k_{m}}{2^{m}}, \frac{k_{m}+1}{2^{m}}\right) \cap S\right)}{\frac{1}{2^{m}}} .
$$

Therefore, from the above inequalities we obtain that $F^{\prime}\left(t_{0}\right) \geqslant \mu(S)$. This is a contradiction, because by Lebesgue differentiation theorem $F^{\prime}\left(t_{0}\right)=0$.

We have showed that the coalition $I$ of all agents blocks $\omega$ via the allocation $x$, but no other coalition, with measure less than 1 , can block $\omega$ via the same allocation $x$. However, we notice that, for any $\varepsilon$, the coalition $\left(\frac{1}{2}-\varepsilon, \frac{1}{2}+\varepsilon\right)$ blocks $\omega$ via the allocation $x^{\prime}$, given by $x_{j}^{\prime}=x_{j}$ if $j \leqslant 3$ and $x_{j}^{\prime}=\omega_{j}$ if $j>3$.

We also notice that $x$ is a Walrasian allocation. In fact, $(x, p)$ is a Walrasian equilibrium if for $p \in \ell_{1}$ there exists $\lambda>0$, such that $p_{j}=\lambda\left(\alpha(j) / x_{j}\right)$. Therefore, $x$ belongs to the core of the economy.

Remark 5. Note that the preference relation in the above example, defined by the utility function $U(x)=\sum_{j \geqslant 1} \alpha(j) \log \left(\frac{1}{2}+x_{j}\right)$, is Mackey continuous, strictly convex, strictly monotone and even myopic, but the exact strong version of Schmeidler's [23] result does not hold.

It is easy to see that example 1 remains true in any Banach lattice containing an unconditionally basic sequence. For this, it is enough to take $C(j)$ satisfying $\sum_{j>0} C(j)<\infty$. In particular, such example remains true in the space $C_{0}$ of all convergent sequences.

EXAMPLE 2. This example shows that if preferences are not Mackey continuous, then the thesis of theorem 1 does not hold.

Let $\mathbb{Q} \cap(0,1)=\left\{q_{n}, n \in \mathbb{N}\right\}$. For each $n$ let $m=m(n)$, such that $I_{n}^{m}=$ $\left(q_{n}-\frac{1}{m}, q_{n}+\frac{1}{m}\right) \subset[0,1]$. Consider the set of intervals $\left\{I_{n}^{m}, n \in \mathbb{N}\right.$, $m \geqslant m(n)\}=\left\{I_{k}, k \in \mathbb{N}\right\}$. By induction, for each $k$ we take a set $H_{k} \subset I_{k}$, with $\mu\left(H_{k}\right)>0$ and $H_{k} \cap H_{k^{\prime}}=\varnothing$, for all $k \neq k^{\prime}$. Note that, for example, we can take $H_{k}$ as a positive measure compact Cantor set included in $I_{k}$, with $H_{k} \cap H_{j}=\varnothing$ for all $j<k$. Observe that, in this case, $I_{k} \backslash \bigcup_{i=1}^{k-1} H_{i}$ is an open set which contains some open interval. Let $A=I \backslash \bigcup_{k=1}^{\infty} H_{k}$. Let $\left\{B_{k}, k \in \mathbb{N}\right\}$ be a family of disjoint sets, such that $\mathbb{N}=\bigcup_{n=1}^{\infty} B_{k}$, and $\operatorname{Card}\left(B_{k}\right)=\infty$ for all $k$.

Now the endowments and preference relation of each agent $t \in I$ are defined as follows: 
If $t \in H_{k}$, then

$$
\omega_{j}(t)= \begin{cases}1 & \text { if } j \notin B_{k} \\ 1+\frac{1}{\mu\left(H_{k}\right)} & \text { if } j \in B_{k}\end{cases}
$$

And for every $t \in A, \omega_{j}(t)=1$, for all $j$.

Every agent has the same preference relation, given by the utility function

$$
U(x)=\liminf _{j}\left\{x_{j}\right\}
$$

We remark that the utility function $U$ is not Mackey continuous on $\ell_{+}^{\infty}$. To see this, let us consider the sequence $x_{n}$ given by $\left(x_{n}\right)_{j}=0$ if $j \leqslant n$ and $\left(x_{n}\right)_{j}=1$ if $j>n$. Then, $x_{n}$ converges to zero with respect to the Mackey topology (see Aliprantis and Border [1, Corollary 13.29]). Finally, notice that $U\left(x_{n}\right)=1$ for each $n$ while $U(0)=0$.

It is clear that $U(\omega(t))=1$ for all $t \in I$. It is also clear that the coalition $I=[0,1]$ blocks $\omega$ via the allocation $x$, given by $x_{j}(t)=2$ for all $j$ and for all $t$.

Suppose now that a coalition $S$ blocks $\omega$. Then $\mu\left(S \cap H_{k}\right)>0$ for all $k$. This implies that $\bar{S}=I$, that is, $S$ is a dense subset of $I$. To show this it is enough to prove that $q_{n} \in \bar{S}$, for all $n$. For this, take $q_{n}$ and any $m \geqslant m(n)$. Let $I_{k}=\left(q_{n}-\frac{1}{m}, q_{n}+\frac{1}{m}\right)$. It is obvious that $S \cap I_{k} \neq \varnothing$. Therefore, if $S \subset \bigcup_{i=1}^{N} S_{i}$, then $\sum_{i=1}^{N} \operatorname{diam}\left(S_{i}\right) \geqslant 1$, and so the result stated in theorem 1 does not hold for this economy.

Remark 6. For the economy stated in Example 2, if a coalition $S$ blocks $\omega$, then $S$ is a dense subset of $I$. Therefore, if preferences are not Mackey continuous, then neither theorem 1 or weaker versions of it remain true. Despite this, note that, for any $\varepsilon>0$, if $H_{k}(\varepsilon) \subset H_{k}$ and $\mu\left(H_{k}(\varepsilon)\right)=$ $\varepsilon \mu\left(H_{k}\right)$, then the coalition $H(\varepsilon)=\bigcup_{k} H_{k}(\varepsilon)$ blocks $\omega$ via the same allocation $x$, and $\mu(H(\varepsilon)) \leqslant \varepsilon$.

\section{APPENDIX}

We collect some results which are particularly helpful in this paper. For this, let us consider the dual pair $\left(\ell^{\infty}, \ell_{1}\right)$.

First, we recall the Schur property notion for a Banach space.

Definition. A Banach space $X$ has the Schur property provided that when a sequence $x_{n}$ converges weakly to 0 , then $\left\|x_{n}\right\|$ converges to 0 (or 
equivalently, if the collections of weakly compact and norm compact subsets of $X$ coincide).

THEOREM (Banach). The Banach lattice $\ell_{1}$ has the Schur property. (See Aliprantis and Border [1, p. 440])

COROLlary. The Mackey topology and the weak star topology coincide on bounded subsets of $\ell^{\infty}$.

Proof. Let $x_{n}$ be a sequence which converges to $x$ with respect to the weak star topology on $\ell^{\infty}$. Let $y_{n}$ be a sequence which converges to $y$ with respect to the norm topology on $\ell_{1}$. Then, $\left\langle\cdot, y_{n}\right\rangle$ converges to $\langle\cdot, y\rangle$ uniformly on bounded subsets of $\ell^{\infty}$. This implies (see Royden [22, Problem 9.40]) that the function $\left\langle x_{n}, \cdot\right\rangle$ converges continuously to $\langle x, \cdot\rangle$ on norm compact subsets of $\ell_{1}$, and therefore, $\left\langle x_{n}-x, \cdot\right\rangle$ converges to zero uniformly on norm compact subsets of $\ell_{1}$ (by the same result in [22]). Therefore, by Banach's theorem, we conclude that $x_{n}$ converges to $x$ with respect to the Mackey topology on $\ell^{\infty}$.

Q.E.D.

Using the corollary above and the separability of the weak star topology, we can take a countable cover of $\ell^{\infty}$ by bounded sets and infer the separability of the Mackey topology on $\ell^{\infty}$.

\section{REFERENCES}

1. C. D. Aliprantis and K. C. Border, "Infinite Dimensional Analysis," Springer-Verlag, Berlin/Heidelberg, 1994.

2. R. M. Anderson and W. R. Zame, Edgeworth's conjecture with infinitely many commodities: L $L^{1}$, Econometrica 65 (1997), 225-273.

3. R. M. Anderson and W. R. Zame, Edgeworth's conjecture with infinitely many commodities: commodity differentiation, Econ. Theory 11 (1998), 331-377.

4. A. Araujo, Lack of Pareto optimal allocations in economies with infinitely many commodities: The need for impatience, Econometrica 53 (1985), 455-461.

5. R. J. Aumann, Markets with a continuum of traders, Econometrica 32 (1964), 39-50.

6. T. Bewley, Existence of equilibria in economies with infinitely many commodities, J. Econ. Theory 4 (1972), 514-540.

7. T. Bewley, The equality of the core and the set of equilibria in economies with infinitely many commodities and a continuum of agents, Int. Econ. Rev. 14 (1973), 383-393.

8. D. Brown and L. Lewis, Myopic economic agents, Econometrica 49 (1981), 359-368.

9. J. Diestel and J. J. Uhl, "Vector Measures," American Mathematical Society, Providence, RI, 1977.

10. N. Dunford and J. T. Schwartz, "Linear Operators," Part I, Interscience, New York, 1958.

11. N. Gretsky and J. Ostroy, Thick and thin market non-atomic exchange economies, in "Advances in Equilibrium Theory" (C. D. Aliprantis, O. Burkinshaw, and N. J. Rothman, Eds.), Lecture Notes in Economics and Mathematical Systems, Vol. 224, Springer-Verlag, Berlin, 1991. 
12. B. Grodal, A second remark on the core of an atomless economy, Econometrica 40 (1972), 581-583.

13. O. Hart, Monopolistic competition in a large economy with differentiated commodities, Rev. Econ. Stud. 46 (1979), 1-30.

14. C. Hervés, E. Moreno, and M. Páscoa, Manipulation-proof equilibrium in atomless economies with commodity differentiation, Econ. Theory 14 (1999), 545-563.

15. L. Jones, Existence of equilibria with infinitely many customers and infinitely many commodities: a theorem based on models of commodity differentiation, J. Math. Econ. 12 (1983), 119-139.

16. A. Mas-Colell, A model of equilibrium with differentiated commodities, J. Math. Econ. 2 (1975), 263-295.

17. A. Mas-Colell, On the continuous representation of preorders, Int. Econ. Rev. 18 (1977), 509-513.

18. A. Mas-Colell and W. R. Zame, Equilibrium theory in infinite dimensional spaces, in "Handbook of Mathematical Economics" (W. Hildenbrand and H. Sonnenschein, Eds.), Vol. IV, Chap. 34, pp. 1835-1898, North-Holland, Amsterdam, 1991.

19. C. Núñez, El teorema de Liapunov en el mecanismo del veto, Revista de la Real Academia de Ciencias Exactas, Físicas y Naturales, de Madrid, Tomo LXXXVII (1993), Cuaderno Segundo-Tercero.

20. J. M. Ostroy, Representation of large economies: The equivalence theorem, presented at the Winter meeting of the Econometric Society, 1973.

21. J. M. Ostroy and W. R. Zame, Nonatomic economies and the boundaries of perfect competition, Econometrica 62 (1994), 593-633.

22. H. Royden, "Real Analysis," Macmillan, New York, 1968.

23. D. Schmeidler, A remark on the core of an atomless economy, Econometrica 40 (1972), 579-580.

24. A. Wilansky, “Topology for Analysis,” Krieger, Malabar, FL, 1970. 